\title{
LA LABOR DE VERIFICACIÓN DE NOTICIAS DESDE EL DEPARTAMENTO DE COMUNICACIÓN. ESTUDIO DE CASOS EN EL SECTOR AGROALIMENTARIO
}

\section{THE WORK OF VERIFICATION OF NEWS FROM THE COMMUNICATION DEPARTMENT. A CASE STUDY IN THE AGRI-FOOD SECTOR}

\author{
Paula Vázquez Almendros. Universidad de Málaga. España \\ paulava@uma.es
}

Francisco Javier Paniagua Rojano. Universidad de Málaga. España fjpaniagua@uma.es

\section{Cómo citar el artículo:}

Vázquez Almendros, P. y Paniagua Rojano, F. J. (2022). La labor de verificación de noticias desde el departamento de comunicación. Estudio de casos en el sector agroalimentario. Revista de Ciencias de la Comunicación e Información, 27, 1-23. https://doi.org/10.35742/rcci.2022.27.e238

\section{RESUMEN}

El presente trabajo analiza la importancia de la verificación de noticias e informaciones desde los departamentos de comunicación de empresas y organizaciones, para combatir la desinformación y los bulos que, bien desde fuera -la competencia, clientes y otros agentes- 0 desde dentro, se generan con diferentes intenciones. El objetivo de esta investigación se centra en definir la organización, la estructura, las herramientas, los planes de acción para combatir estos bulos, rumores y la desinformación sobre empresas e instituciones. Para ello, esta investigación se basa en el estudio de caso de la cooperativa Dcoop. En concreto, partiendo de la observación directa, el análisis de contenido y el cuestionario a socios y empleados de la sociedad se pretende diseñar una estrategia para luchar contra la desinformación y los bulos, con la implicación de los diferentes grupos de interés. Como solución se propone reforzar la estrategia de comunicación con su complicidad y una propuesta de organización y protocolo de acción desde el área de Comunicación de la empresa, reforzando también la comunicación permanente y la transparencia.

PALABRAS CLAVE: comunicación estratégica, comunicación corporativa desinformación, comunicación interna, DIRCOM.

\section{ABSTRACT}

This paper analyzes the importance of the verification of news and information from the communication departments of companies and organizations, in order to combat disinformation and hoaxes that are generated with different intentions, either from outside -competitors, clients and other agents- or from within. The objective of this research focuses on defining the organization, structure, tools and action plans to combat these hoaxes, rumors and disinformation about companies and institutions. To 
this end, this research is based on the case study of the Dcoop cooperative. Specifically, based on direct observation, content analysis and a questionnaire to members and employees of the company, the aim is to design a strategy to fight against disinformation and hoaxes, with the involvement of the different stakeholders. As a solution, it is proposed to reinforce the communication strategy with their complicity and a proposal of organization and action protocol from the Communication area of the company, also reinforcing permanent communication and transparency.

KEYWORDS: strategic communication, corporate communication, disinformation, internal communication, DIRCOM.

\section{INTRODUCCIÓN}

En este contexto; con la llegada de Internet y la web 2.0, en el que las organizaciones han perdido el control sobre su discurso público (Losada, 2018), y la empresa ya nunca volverá a ser la única emisora de información sobre la marca y el proceso comunicativo no volverá a ser unidireccional" (Aced, 2013, p.18); la gestión de la comunicación en situaciones de crisis se ha instalado en el quehacer diario de las empresas y las organizaciones (Álvarez, 2009).

En este nuevo panorama mediático, "la marca es la expresión de un compromiso de la empresa con sus stakeholders, una especie de promesa hacia estos" (Villafañe, 2004 , p. 39) y, en este nuevo sistema, en el que ya no solo los medios de comunicación tradicionales acaparan todo el protagonismo, existen nuevos actores que tienen el poder de coproducir, tanto positiva como negativamente, la reputación que proyecta una entidad a sus públicos.

La llegada de la web 2.0 y el uso de las redes sociales han supuesto un cambio de mentalidad respecto a la manera de comunicar. El papel de emisor y receptor ha pasado a ser intercambiable entre los diferentes actores que intervienen en el sistema comunicativo y esa comunicación, ahora, está basada en la conversación (Aced, 2013). "El público abandona su estado de pasividad y ahora se piensa protagonista" (Álvarez, 2012).

Según recoge el Edelman Trust Barometer 2021, durante el pasado año la confianza depositada en los medios de comunicación y las informaciones que reciben desde sus gobernantes han descendido. Uno de los datos más relevantes del estudio es que, en 18 de los 27 países analizados, las empresas cuentan con mayor índice de confianza en la información que emiten que el propio Gobierno. Ahora bien, en este panorama, en el que la empresa se sitúa como fuente fiable de información ante la ciudadanía, estas deben adoptar nuevas medidas de compromiso con sus públicos y adaptar nuevas estrategias de comunicación en las que destaquen la transparencia y la difusión de información veraz, y centrarse en solucionar las preocupaciones que afectan a sus grupos de interés y a enfrentar la lucha contra la desinformación con ayuda de esos mismos stakeholders.

Un papel muy relevante dentro de la difusión de la desinformación y los bulos recae sobre las emociones. La importancia que poseen las emociones sobre el público es 
enorme, ya que estas se encuentran presentes en la interpretación de los hechos que pueden provocar una crisis, a la vez que en la interpretación que los diferentes públicos hacen sobre la responsabilidad de esos hechos junto a la respuesta que desde la organización afectada se adopta en cada momento. (Losada et al., 2020).

Son muchos los ejemplos en los que la desinformación las noticias falsas y los rumores han afectado a las empresas de todos los sectores. A lo largo de los años han existido diversas crisis muy graves dentro del sector. Ejemplo de ello fue la sufrida en 1981 con el llamado Aceite Tóxico. Después, han sido muchas otras las que han puesto en jaque al sector de la alimentación y la bebida, las conocidas "vacas locas", las diferentes crisis de Coca-Cola, la gripe aviar, y otras más actuales como el caso de Danone. Y en todos estos casos, el daño que una noticia falsa puede provocar es mayor, ya que la credibilidad de la imagen se basa en unos productos saludables.

Cada vez son más frecuentes los tipos de crisis que se ocasionan por la desinformación y las noticias falsas. Este tipo de informaciones deriva del cambio en el consumo de información. De esta forma, Wardle (2017) clasifica las desinformaciones en sátira o parodia, contenido engañoso, contenido impostor, contenido fabricado, conexiones falsas, contexto falso y contenido manipulado. Esta clasificación se puede completar, en el caso de las relaciones públicas, con la propuesta por Rodríguez-Fernández (2019) que propone el estudio de la identificación de instalaciones ajenas a las compañías, ofertas falsas de empleo, usurpación de la identidad, regalo de producto/servicio y promociones falsas, búsqueda de influencers por parte de la marca, falsos accidentes, y publicación de desinformación y falsas portadas en medios de comunicación generalistas.

El por qué este tipo de informaciones puede tener un tan alto nivel de repercusión en la reputación de las empresas viene condicionado por la facilidad con la que se difunden a través de los nuevos medios y las redes sociales. Algunos estudios coinciden en que las noticias falsas son más novedosas que las verdaderas, por lo que la gente se siente más atraída por ellas y se encuentra más dispuesta a compartirlas (Vosoughi et al., 2018). Pero, debemos tener presente que la desinformación en línea no es solo un fenómeno impulsado por la tecnología (Humprecht, 2019), también se encuentra condicionado por los entornos nacionales de la información. Cabe destacar que una gran parte de los artículos publicados por los sitios de noticias falsas se parecen mucho a las noticias tradicionales (Tandoc Jr. et al., 2021) y es que la mayoría de esos artículos contienen valores noticiosos como la actualidad o la negatividad, donde las emociones juegan un papel fundamental (Losada et al., 2020).

Otro factor, cada vez más común al que se enfrentan las empresas que sufren ataques basados en la desinformación, se centra en que, muchas veces, no se sabe quién se encuentra detrás por lo que resulta difícil poder llegar a combatirlos (Sánchez-Silva, 2019). El informe Industrialized Disinformation 2020 Global Inventory of Organized Social Media Manipulation, asegura que existen cibertropas que se encargan de llevar a cabo estrategias de comunicación entre las que se incluye la creación de sitios web de las llamadas "noticias falsas". 
De este estudio se extraen las cuatro categorías de actividades que desarrollan este tipo de perfiles; creación de contenidos manipulados; crear estrategias definidas para dirigirse a segmentos concretos de la población; el acoso en línea o trolling y, por último, la censura de contenidos por medio de denuncias masivas. "La desinformación es un negocio para agencias que se dedican a ofrecer servicios de desinformación, para organizaciones que se dedican a certificar contenidos a través del fact-checking y para investigadores que estudian la desinformación y buscan soluciones". (FrancoisFogel, 2020).

Por todo esto, resulta imprescindible que las empresas sean proactivas y se posicionen en la batalla contra la desinformación con un plan estratégico bien definido. Contar con un plan de comunicación, que recoja una política de comunicación para cada crisis. La transparencia en la difusión de los contenidos, la disponibilidad de atender a los medios y decir siempre la verdad, son las señales de una política de comunicación proactiva que debe englobarse dentro de un plan de prevención de crisis de toda empresa. Además, el desarrollo de internet para la gestión de crisis muestra una gran potencialidad en la estrategia de ser proactivos (Rodríguez González et al., 2013).

El hecho de ser proactivos facilitará la tarea de enfrentarse a las crisis ocasionadas, cada vez más, por la desinformación y los rumores. Del estudio de Llorente \& Cuenca (2018) se extrae que la transparencia y la detección temprana del bulo son clave para combatir este tipo de crisis. Realizar una escucha activa en redes sociales, actuar y desmentir la información falsa y, por último, responder en la misma dirección que el contenido engañoso. Además, es importante trabajar en la prevención de este tipo de informaciones, humanizar la comunicación; potenciar el liderazgo de la empresa; posicionar a los embajadores de la marca, de forma que transmitan fielmente la realidad de la compañía y a la vez sean una defensa ante los bulos y contar siempre con la transparencia para crear una buena reputación.

Así, la escucha activa es fundamental para conseguir una comunicación pública ética. Es necesario usar la comunicación bidireccional para ser éticos y conseguir una influencia mutua. No se puede ser influenciado por un grupo si no se escucha (Coombs and Holladay, 2007, como se citó en Macnamara, 2016). Y es que, la comunicación bidireccional no es solo cada vez más demandada por los públicos, sino que es de suma eficiencia en las organizaciones a la hora de escuchar y responder a las perspectivas más amplias que son clave para hacer operativa la responsabilidad social de las empresas (Macnamara \& Gregoru, 2018).

Del European Communication Monitor (2018) se extrae que un 22,5\% de las organizaciones europeas se han visto afectadas por noticias falsas, de las cuáles las principales fuentes de difusión de dicho contenido fueron las redes sociales. De todas ellas, solo el $12 \%$ ha implantado una serie de medidas para identificarlas. La mayoría de profesionales encuestados en este mismo estudio, pero elaborado dos años después, European Communication Monitor (2020), reconoce que los nuevos canales de comunicación digital traen consigo nuevos desafíos éticos, pero confiesa que no cuentan con los recursos actualizados para abordarlos. 


\subsection{Las nuevas funciones del DIRCOM}

A lo largo de los años, la figura del Director de Comunicación se ha ido fortaleciendo y haciéndose más imprescindible en todo tipo de empresas y organizaciones. Con la llegada de la web 2.0 e Internet, el modelo de comunicación ha sufrido un cambio que exige al DIRCOM nuevas responsabilidades. Según el Anuario Dircom (2014), entre las nuevas funciones se recoge la actualización de la web corporativa, comunicación de carácter recurrente a través de internet, la gestión de los blogs de la corporación y el control y presencia en todo tipo de redes sociales como Twitter, Facebook o Linkedin, entre otras. Es así que Taddicken \& Wolff (2020) demostraron la importancia que conlleva tener un nivel adecuado de conocimiento sobre los medios e Internet.

La figura del nuevo profesional de la comunicación debe estar dispuesta a adquirir las competencias que se demandan en el nuevo panorama informativo. Junto a esas nuevas funciones ya mencionadas, Ufarte-Ruiz et al., (2018) recogen que, otras de las competencias que todos estos perfiles deben adquirir, debido a la llegada de ese sistema digital en el que todo está conectado, es la formación en torno a la lucha contra la desinformación y el desarrollo del fact cheking. "La evolución de iniciativas de verificación de hechos se ha desarrollado de forma acelerada en los últimos años" (Magallón, 2018, p. 45).

Esto se debe a que son estos profesionales los que deben establecer y reforzar estrategias que se centren en la formación de los usuarios para desarrollar un uso adecuado de la información y la correcta validación de las distintas fuentes. Esto permitirá llegar a una serie de proyectos que se centren en la formación de competencias digitales y mediáticas (A. López, \& J. Vives, 2018).

\subsection{El papel de los públicos internos como embajadores de la marca}

"La reputación es la cristalización de la imagen corporativa de una entidad cuando ésta es el resultado de un comportamiento corporativo excelente, manteniendo a lo largo del tiempo, que le confiere un carácter estructural ante sus stakeholders estratégicos" (Villafañe, 2004, pp. 32-33). Es importante tener en cuenta que, en cualquier organización, el papel del primer stakeholders lo componen los propios empleados. Estos, al igual que los socios, los clientes, los accionistas o proveedores son considerados como un grupo cuya opinión debe ser valorada a la hora de analizar la reputación de una organización. Este hecho debe ser contemplado por todas las empresas y organizaciones ya que la actuación reputacional que llevan a cabo esos empleados tiene su reflejo en muchos aspectos como pueden ser las redes sociales, su comportamiento en el trabajo o en sus interacciones personales. Por ello, se debe asegurar que este grupo de interés dispone de los conocimientos, criterios y motivos necesarios que les permitan defender y explicar la posición de la empresa. (Estudio de comunicación, 2020). "El futuro de las empresas supondrá cada vez una mayor interconexión de quienes trabajan en ellas" (Vilanova, 2013, pp. 118).

Es por ello por lo que los planes de comunicación de las empresas establecen a las personas encargadas de hablar en nombre de la organización cuando se da una situación de crisis, pero, se debe tener en cuenta que, todos los empleados son portavoces de carácter informal de esa compañía. Y es que, en la sociedad actual, 
multiconectada y online, lo que los empleados de una empresa transmiten obtiene más credibilidad que lo que la propia compañía pueda ofrecer en declaraciones oficiales. (Estudio de Comunicación, 2020).

\section{OBJETIVOS}

El presente trabajo tiene como objetivos principales proponer la creación de una unidad de verificación de noticias e informaciones dentro de los gabinetes de comunicación de empresas y organizaciones en el sector agroalimentario; y la definición de la estrategia que deben seguir las empresas y organizaciones en estos casos, así como las principales herramientas que pueden utilizar, y qué pasos se deben de dar y cómo implicar a los diferentes stakeholders en la coproducción de la reputación de dicha organización. En este sentido, se plantean, como objetivos complementarios:

- Definir los indicadores necesarios para llevar a cabo un análisis completo de los diferentes casos de crisis

- Identificar diferentes herramientas de verificación de la información

- Identificar a los grupos de interés que puedan convertirse en aliados del departamento de comunicación en la estrategia de combatir los rumores y la desinformación contra la compañía

- Fortalecer la escucha activa con los diferentes stakeholders

- Proponer herramientas de comunicación internas y externas para fortalecer la comunicación con los grupos de interés y reforzar la credibilidad de la organización

\section{METODOLOGÍA}

Este trabajo parte de la hipótesis de que, las empresas del sector agroalimentario, como cualquier otra, deben hacer frente a las nuevas crisis digitales, basadas en la desinformación y las noticias falsas; desde un punto de vista ético, incentivando la transparencia e implicando a todos sus stakeholders, como embajadores y portavoces en las redes sociales y en los medios de comunicación. Así reducirán el impacto de estas crisis en su reputación, que debe ser trabajada a diario, y no solo si situaciones críticas y de riesgo.

Dentro de las ciencias sociales, y más concretamente dentro del periodismo y la comunicación social, la investigación científica se aborda desde dos perspectivas metodológicas; la metodología cuantitativa y cualitativa. Cada una presenta unas características acordes a los diferentes objetos de estudio, pero no se trata de métodos excluyentes sino complementarios (Monje Álvarez, 2011). En el presente trabajo se ha llevado a cabo una recogida de información por medio de técnicas metodológicas cuantitativas, en las que se recogen y analizan datos y se sirven de la medición numérica para establecer patrones entre la muestra estudiada, y técnicas cualitativas, que permitan definir y descubrir preguntas de investigación sin basarse en la medición numérica, como la observación y la descripción (Hernández et al., 2004).

Esta investigación, se basa en el estudio de caso, como técnica que permite realizar una recopilación e interpretación de toda la información sobre un individuo, empresa 
o movimiento (Reyes, 1999). Según Yin (1994) los estudios de caso son: las estrategias preferidas cuando las preguntas "cómo" y "por qué" son realizadas, cuando el investigador tiene poco control sobre los eventos, y cuando el foco está en un fenómeno contemporáneo dentro de un contexto de la vida real (p.2).

Los estudios de caso pueden clasificarse en dos tipos según Reyes (1999). El primero intenta derivar conclusiones generales que se extraen del análisis de una serie de casos y el segundo cuando se pretende llegar de forma específica a unas conclusiones de un único caso.

Los elementos que se deben de tener en cuenta a la hora de diseñar el estudio de casos son cinco. En un primer lugar el estudio de caso debe de contar con una pregunta de estudio, esta será la base del estudio de caso; en segundo lugar se encuentra el estudio de las proposiciones, es decir, la proposición que va a ser examinada y sobre la que se dirige la atención, sin embargo no todos los estudios tienen que contar con este elemento; el tercer componente es la unidad o unidades de análisis y, por último, la lógica que une los datos y el criterio por el que se interpretan los resultados (Yin, 1994).

Varios autores reconocen que el estudio de caso puede presentar una dualidad que se caracterice como problemática. Por un lado, el estudio de caso expresa tanto lo particular como lo específico y a su vez puede implicar a una población y llegar a aludir a otros casos. Estos factores desembocan en que los hallazgos vayan mucho más allá de los límites del propio investigador (Forni, 2010).

El estudio de caso de este trabajo se centra en la cooperativa DCOOP, para llevarlo a cabo se han establecido una serie de variables de carácter analítico de acuerdo con las referencias ya mencionadas, con el objetivo de estudiar cómo se puede definir la estructura, las herramientas y las diferentes acciones para combatir bulos, rumores y desinformación desde los diferentes gabinetes de comunicación de empresas y organizaciones y qué perfil profesional debe tener la persona que lo gestione.

En primer lugar, se parte de una observación directa participante, motivada por las prácticas profesionales, llevadas a cabo en la cooperativa de segundo grado DCOOP por parte de uno de los autores de la investigación. Este hecho permite conocer desde dentro el funcionamiento de la cooperativa, su departamento de comunicación, estrategias que llevan a cabo, manejo de la página web, uso de las redes sociales, etc., que sirvió para definir el estudio de caso.

Para completar la metodología del trabajo, se desarrolla un análisis de contenido, que se centra en establecer un objeto de estudio para cuya representación usa la selección y el análisis de elementos comunicativos (Piñuel Raigada, 2002) para estudiar el caso concreto de Dcoop y las noticias falsas publicadas en algunos medios durante 2018, y al mismo tiempo valorar su estrategia ante estos casos y en circunstancias normales. Según Krippendorff (1980) y Lozano (1994) esta técnica de investigación facilita la adquisición de nuevos conocimientos, aumenta la comprensión del investigador sobre el tema e informa sobre las acciones prácticas. En concreto, se analizaron 51 noticias publicadas en diversos medios digitales, generalistas, económicos y especializados del sector - Agrodigital, Agroinformación, Agropopular, Alimarket, Almaceite. Crónica 
Global, Deoleo, Diario de Jaén, El Economista, El País, El Plural, Expansión, Ideal, La otra Andalucía, La Vanguardia, Mercacei, Merca2, Olimerca, Okdiario, Servimedia, Viva, Voz Populi-, entre el 3 de junio de 2018 y el 3 de enero de 2019, fechas en las que se publicaron diferentes noticias falsas sobre la empresa objeto de estudio.

Por último, se elaboraron dos cuestionarios a una serie de perfiles relevantes dentro y fuera de la cooperativa -trabajadores y socios con más actividad en las redes sociales-, para conocer su percepción sobre la comunicación de esta y sobre la estrategia que se realiza en redes e implicarlos en la estrategia activa en la lucha contra la desinformación contra la empresa. Gracias a esta técnica, se recogieron datos sobre una población concreta de la empresa -socios y empleados con presencia y actividad en las redes sociales-, a pesar de las dificultades para reunir a los sujetos, ya sea por la distancia o la dispersión de estos (García, 2003). Las preguntas se presentan de forma organizada, y se centran en un caso particular, el de Dcoop, su estrategia en redes sociales y en la lucha contra la desinformación, y se basa, por tanto, en el análisis y tratamiento de datos obtenidos de un grupo de personas que representan a una población (García, 2003; Meneses \& Rodríguez, 2011).

\section{DISCUSIÓN}

\subsection{Estudio de caso. Crisis de bulos y desinformaciones sufridas por DCOOP en 2018}

El estudio de caso, tal y como se ha mencionado en el objeto de este trabajo, se ha centrado en el análisis de un conjunto de noticias publicadas desde junio de 2018 hasta diciembre del mismo año cuyo objetivo era dañar la reputación de la cooperativa. En total, se han analizado 74 noticias que dañaban la reputación de la cooperativa al contener información inexacta o falsa.

Conocer la trayectoria de la cooperativa es necesario para poder analizar la situación en la que se encontraba DCOOP en el momento en el que su reputación se vio gravemente dañada por la publicación de una serie de desinformaciones. Durante 2017, la cooperativa había alcanzado el $50 \%$ de participación en la marca Pompeian, marca con la que comercializa en EEUU, también había realizado la fusión con la Orujera Interprovincial de Fuente de Piedra junto a la creación de las secciones de frutos secos, industrial de leche y orujo y se había producido la integración de nuevos socios internacionales y de sociedades del grupo De Prado en Córdoba, Chile y Portugal.

En 2018, la cooperativa adquirió participaciones en empresas aceituneras de marca líder en EEUU (Bell-Carter) y Portugal (Maçarico) e inauguró el nuevo laboratorio de aceite junto a la puesta en marcha de la almazara de Dos Hermanas.

Del análisis realizado se extraen una serie de conclusiones. En primer lugar, se han clasificado en grupos los diarios de los que procedían las 74 informaciones analizadas en el estudio de caso. Así es que, un $44,59 \%$ de los contenidos analizados procedían del mismo medio, en concreto del diario digital Crónica Global, destaca que las noticias que este medio publicaba eran compartidas posteriormente por otros diarios. 
A un segundo grupo pertenecen los diarios Merca2 y EIPlural de los que procedían el $12,16 \%$ de los contenidos. También destaca el caso de los diarios Revista Almaceite, OKDiario y Diario de Jaén, el porcentaje de contenidos publicados por cada uno de ellos asciende al $4,05 \%$ del total, junto al $2,7 \%$ de las publicadas por Servimedia, El País o Voz Populi. Un último grupo de noticias, que representa el $1,35 \%$ de los contenidos analizados, procedía de los diarios Olimerca, Alimarket, Viva, El Expansión y el Expansión.

Cabe destacar que en la mayoría de las noticias analizadas predominan las fuentes anónimas $(66,21 \%)$. En un $12,16 \%$ de los contenidos la fuente que suministra la información es otro medio de los analizados y en un $9,45 \%$ se recogen como fuentes las declaraciones de personajes públicos del sector.

Es importante resaltar que, entre las noticias analizadas, un $12,16 \%$ se basa en declaraciones procedentes de DCOOP, ya sean del presidente o de algún otro cargo de la cooperativa. En todos los casos las declaraciones procedentes de la sociedad se centran en defender la reputación de la sociedad ante las falsas informaciones que se difunden sobre ella.

Dentro de las variables de carácter analítico del estudio de caso se analizó también la temática, tanto general como específica, en la que se centran las noticias publicadas. Al referirnos a temática general destacan dos temas predominantes, la compra y venta de aceite $(94,59 \%)$ y la producción y distribución de este $(5,4 \%)$. Por su parte, y en lo que se refiere al nivel individual de cada una de las informaciones, del análisis se desprende que un $50 \%$ de los contenidos publicados se centran en declarar que desde la cooperativa se están llevando a cabo prácticas que banalizan el sector para provocar la caída de precios y conseguir establecer un monopolio. En segundo lugar, un $21,62 \%$ de las informaciones recogen una multa impuesta a la cooperativa por la importación de aceite de países extracomunitarios, un $17,56 \%$ se centra en la importación de aceite de países como Marruecos o Túnez para venderlo como producto español, mientras que un $13,51 \%$ habla sobre la intención de la cooperativa para incentivar la creación de aranceles en EEUU. 


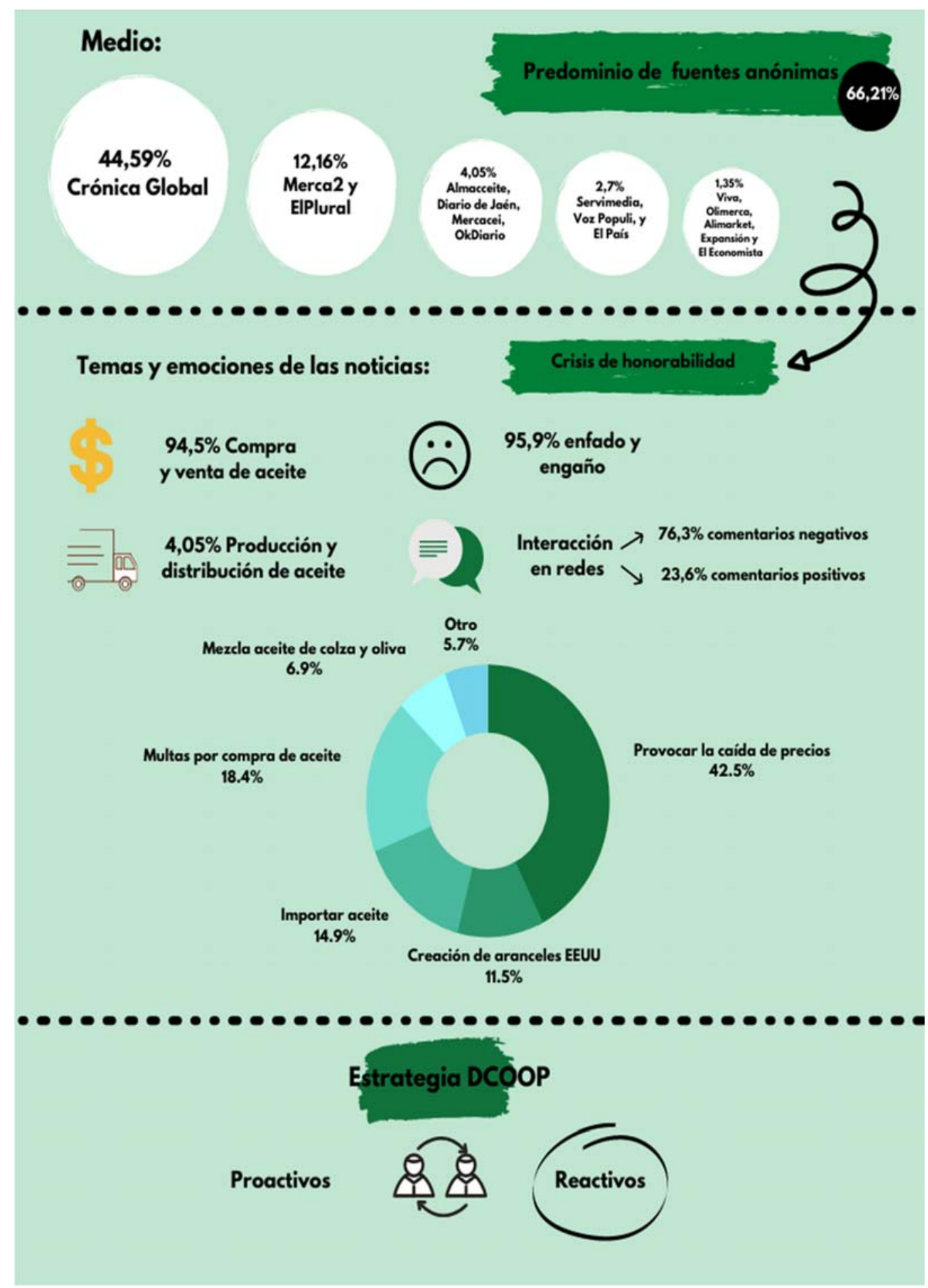

Figura 1. Análisis de los bulos publicados en diferentes medios sobre Dcoop en 2018. Fuente: análisis de noticias falsas sobre la empresa. Elaboración propia

El resto de las noticias analizadas recogen una variedad de temas menos representativo, el más destacado de ellos es la mezcla de aceite de oliva y aceite de colza y su venta en el mercado americano $(8,1 \%)$. 
Otra de las variables analizadas se basa en la clasificación de Wardle (2017) sobre los diferentes tipos de fake news o desinformaciones que se difunden en el sistema digital, dentro del estudio de caso se ha identificado que el $86,48 \%$ del contenido publicado corresponde a una desinformación de contexto falso. Esto quiere decir que se está ofreciendo contenido veraz, pero rodeado de una información de contexto falsa. Por otro lado, el $12,16 \%$ de las noticias restante se clasifican, siguiendo con la tipología de esta autora, como contenido fabricado, cuyo objetivo y diseño se centra en engañar y hacer daño. Asimismo, Rodríguez (2019) establece una clasificación sobre los tipos de bulos que afectan a las empresas, en este caso, todas las noticias aquí recogidas se encuadran dentro de lo que esta autora refleja como publicaciones de desinformación y falsas portadas en medios de comunicación generalistas.

Una vez analizado el tipo de bulo o desinformación que se ha publicado, es necesario analizar y estudiar el tipo de crisis que esta situación provoca y la repercusión que esta tienen sobre la cooperativa. Según Villafañe (1998), las crisis se clasifican atendiendo a las causas que las originan, es por ello por lo que, y siguiendo la clasificación que propone este autor, el conjunto de las 74 noticias analizadas en este estudio de caso desembocó en una crisis de honorabilidad para la cooperativa, cuya intencionalidad era dañar su reputación y provocar la enemista entre la cooperativa y sus socios.

De la misma forma se ha tenido en cuenta, a la hora de realizar el estudio de caso, la difusión de esas desinformaciones en redes sociales. Gracias a la herramienta de rastreo, CrowdTangle, se pudo contabilizar las veces que estos contenidos fueron compartidos en las redes y el tipo de comentarios que han recibido. Es importante tener en cuenta que este estudio de caso se ha centrado exclusivamente en la red social Facebook, al ser la que ofrecía datos de rastreo suficientes.

Por consiguiente, al contabilizar las veces que las noticias fueron compartidas en esta red social se alcanza un total de 108 post publicados, en los cuales se han registrado un conjunto de 241 comentarios, 184 de ellos negativos y 57 positivos.

Algunos de los comentarios analizados, tanto negativos como positivos:

Esto es lo q le importa a DCOOP...la pasta...Y lo demás a la RUINA...

Es bastante más :3, que los que hay en su cortijo. El y todos los mandamás de DCOOP

Si no fuera por DCOOP veríamos como se vendería el aceite,jugarían con las cooperativas los compradores pagando lo que quisieran,estos artículos son basura

Cuando hay tantos detractores de Dcoop. es la prueba de que lo están haciendo bien. Yo estoy en Decoop. Y esto es falso.

Figura 2. Ejemplos de comentarios en la página de Facebook de Dcoop 
Los comentarios considerados negativos desprestigian la marca y la cooperativa y tienen su base en las noticias analizadas, todo lo atribuyen a ese contenido. En el caso de los comentarios positivos destaca que una gran mayoría alude a la visión crítica que ayude a diferenciar la desinformación de la información veraz y contrastada, otra parte de esos comentarios corresponden a socios de la cooperativa que defiende su reputación como embajadores de marca.

Es importante destacar la importancia que tienen las emociones que se proyectan en las noticias. Así es que en las informaciones analizadas dentro del estudio las emociones a las que se pretende apelar son la tristeza y el enfado, ya que se utiliza un lenguaje negativo y sentencioso.

La última parte del estudio de caso se centra en analizar la respuesta que se ofreció desde la cooperativa DCOOP para hacer frente a este tipo de bulos y desinformaciones que ponían en jaque su reputación.

La sociedad emitió una serie de comunicados internos e incluso se realizó un encuentro con los medios para poder defender la imagen de la cooperativa de las acusaciones a las que estaba siendo sometida. Además, después de la publicación de algunos de estos contenidos, hubo medios que entrevistaron a responsables de la sociedad para conocer y recoger la versión de la cooperativa ante aquellas acusaciones. Ejemplo de ellos fue Mercacei, que publicó el 18 de junio de 2018 una entrevista en la que se recogía la versión de la cooperativa ante aquellas acusaciones.

Algunos ejemplos del uso del lenguaje que se presentaba en esas informaciones.

\section{Confirmado: Dcoop hunde los precios del aceite \\ Dcoop, la aceitera que menos paga a sus olivareros}

está preocupando cada vez más a buena parte de los productores, alarmado por las prácticas de Dcoop, la mayor cooperativa aceitera de

Figura 3. Ejemplos de noticias publicadas en Crónica Global

\subsection{La implicación de los grupos de interés en la estrategia contra la desinformación}

El cuestionario se envió durante la semana del 5 de abril se envió un cuestionario online a socios de la empresa cuyo fin era conocer la visión de estos sobre la información que reciben desde la organización, trabajar en la alineación de la filosofía corporativa y reducir el impacto que la desinformación y los bulos pueden tener en la imagen de la cooperativa y su negocio. En total, el cuestionario se envió a 12 socios de diferentes cooperativas del grupo, que suelen tener actividad en redes sociales, de los cuales contestaron 10 de ellos. El $40 \%$ de las respuestas correspondían a mujeres y el $60 \%$ a hombres, predominando las franjas de edades de entre 35 - 44 y $45-54$ 
años, ambos con el $40 \%$ del total. Seguidos de un $10 \%$ de la franja de edad de $55-$ 64 años y otro $10 \%$ que supera los 65 años.

En primer lugar, el cuestionario pedía a los socios que definieran en tres palabras la figura de la cooperativa. En la siguiente infografía se representan la selección de las palabras elegidas. Destaca Gran Cooperativa, Servicios, Proyecto o Desinformación, entre otras.

Al hablar de la frecuencia con las que los socios dicen informarse sobre Dcoop destaca que un $10 \%$ no suele buscar información sobre la cooperativa nunca y un $30 \%$ dice que sí lo hace, pero una vez a la semana. En contraposición, un $40 \%$ reconoce que lo hace todos los días y un $20 \%$ dos o tres veces a la semana.

Al referirse a la utilidad de la información que reciben por parte de la cooperativa, los socios la valoran con un 6,5 de media sobre 10 . Destaca que un $10 \%$ de los encuestados califica dicha información con un 0 al pensar en la utilidad que les aporta.

En relación con la información que les gustaría recibir por parte de la cooperativa, conocer los logros y avances junto a la historia de esta son los dos tipos de informaciones que más importancia e interés despiertan en los socios, seguidos de la marcha económica, las actividades que puedan afectar a su imagen y la versión de la entidad ante cualquier bulo, desinformación o ataque.

A la hora de analizar los canales por los que la cooperativa informa, las reuniones son la modalidad preferida y mejor valorada entre los socios, su porcentaje de valoración es de un $2,25 \%$ seguido del WhatsApp, newsletter, los medios de comunicación y el cara a cara, cada uno con 1,8 sobre 5 .

De la misma forma, a la hora de seleccionar los canales por los que les gustaría recibir la información que les llega de la cooperativa, las reuniones y el WhatsApp son los dos medios de comunicación más seleccionados con un 90 y un $80 \%$ respectivamente. Por otra parte, es importante tener en cuenta que el $100 \%$ de los socios cuenta con un perfil en Facebook y el $90 \%$ en la red social de WhatsApp. Por el contrario, entre el resto de las canales, solo un $20 \%$ de los socios tienen presencia Twitter y el $10 \%$ en Telegram.

Para saber si los socios se sienten implicados con las informaciones, se les pregunto si comparten información de la sociedad en las redes sociales y un $80 \%$ afirma que sí lo hacen, frente al $20 \%$ que declara no hacerlo. Pero al analizar si cuando comparten esa información en las redes consideran que disponen de todos los argumentos para hacerlo, solo el $33 \%$ considera que sí, frente al $33 \%$ que no y el otro $33 \%$ que considera que tal vez.

Frente a esto, un $80 \%$ de los encuestados dice solicitar información a una fuente oficial de la cooperativa cuando quiere contestar a un mensaje en las redes y considera que no dispone de la información necesaria y suficiente. Cabe destacar que, también a un $80 \%$ de los socios encuestados les gustaría disponer de uno o varios canales por los que realizar consultas y encontrar información. WhatsApp y el correo electrónico son los dos canales preferidos para desarrollar esta función. 
En relación con la pregunta de si alguna vez ha encontrado, en las redes sociales o en algún medio de comunicación, una información falsa o un rumor que dañase la reputación de Dcoop, el $90 \%$ de los encuestados afirma que sí y el $10 \%$ restante declara no recordarlo. En el caso de haberlas encontrado, el 22,2\% confiesa que consultó al departamento de comunicación de la cooperativa frente a otro $22,2 \%$ que afirma que contesto lo primero que pensó en ese momento.

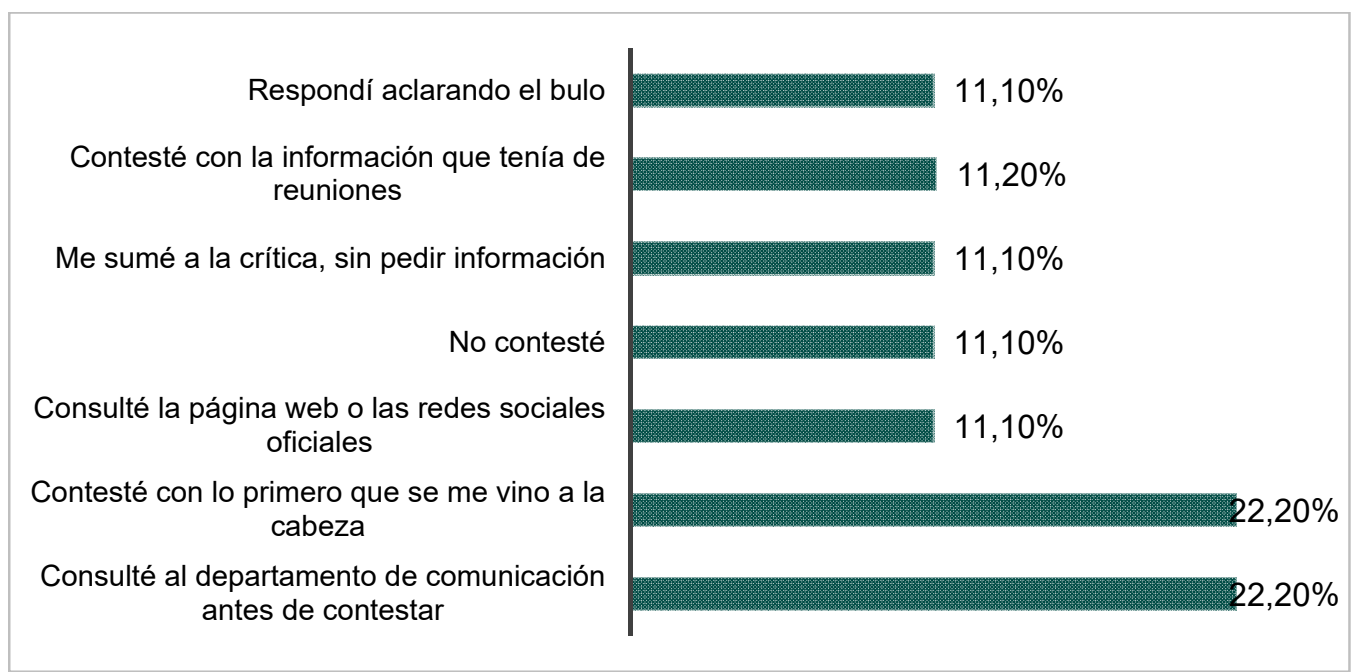

Figura 4. Qué hacen los socios cuando encuentra noticias falsas sobre la empresa.

Fuente: Cuestionario realizado a socios de DCOOP. Elaboración propia

Para el $80 \%$ los socios encontrar un bulo o rumor en las redes o en los medios les afecta de forma directa porque se sienten parte de la cooperativa, por otra parte, a un $70 \%$ les preocupa que afecte a la reputación de la entidad y estarían dispuestos a cambiar esa opinión publicada con argumentos. Destaca que un $10 \%$ afirma que ese tipo de desinformación no les supone ningún problema.

Ante la creciente presencia de bulos y desinformaciones, un $80 \%$ de los socios dice saber reconocer una fake news que quiere dañar la imagen de la cooperativa. Comprobar el medio, la fecha o las fuentes que aparecen en la noticia son los elementos clave que les ayudan a identificar si la información es cierta o no.

De la misma manera, contar con ayuda, por parte de la cooperativa, de toda la información posible para diferenciar ese tipo de desinformación de las noticias reales es algo muy importante para el $60 \%$ de los socios.

A la hora de valorar si consultaría un blog que cuenta con información, formación y tutoriales sobre posibles bulos, rumores e informaciones que dañan la imagen de la sociedad, sobre cómo responder y a la vez conocer el posicionamiento de DCOOP, el $80 \%$ de los encuestado afirma que sí, frente al $10 \%$ que considera que sería mejor un canal más directo y otro $10 \%$ que sí lo haría si se mantiene actualizado y es ágil.

En último lugar, se les pregunto a los socios cómo valorarían de 1 a 5 , siendo 5 la nota más alta, las diferentes acciones que se pueden llevar a cabo desde el departamento 
de comunicación para afrontar cualquier crisis en redes sociales y combatir las noticias falsas y los rumores sobre la sociedad. Trabajar más la reputación de la cooperativa y contar con un protocolo definido internamente son las dos acciones consideradas más importantes.

Del mismo modo, el mismo día del envío del cuestionario a socios, se envió el mismo cuestionario a 15 trabajadores de la cooperativa para conocer la visión de los públicos internos sobre la información que reciben desde la organización, el trabajo en la alineación de la filosofía corporativa y conocer su valoración para reducir el impacto que la desinformación y los bulos pueden tener en la imagen de la cooperativa y su negocio.

En total se recibieron 8 respuestas. En este caso el $25 \%$ de las respuestas corresponde a mujeres y el $75 \%$ a hombres, predominando la franja de edades de entre $35-44$ y 55 - 64 años.

En el caso de los trabajadores, a la hora de definir en tres palabras la figura de la cooperativa, las palabras que más destacan son Confianza, Liderazgo, Estabilidad, Unión y Calidad, entre otras.

En relación con la pregunta sobre la frecuencia con las que los empleados dicen informarse sobre DCOOP, destaca que un $13 \%$ no suele buscar información sobre la cooperativa y otro $13 \%$ dice que sí lo hace, pero una vez a la semana. En contraposición, un $62 \%$ reconoce que lo hace todos los días y un $12 \%$ dos o tres veces a la semana.

Cabe destacar que, a diferencia de los socios, los empleados valoran con una nota de un 7,1 la utilidad de la información que reciben desde la cooperativa. Además, es llamativo que un $12,5 \%$ de los encuestados califican con un 2 la utilidad de esa información.

Al analizar la información que les gustaría recibir por parte de la cooperativa, conocer la versión de la sociedad ante cualquier bulo o ataque y sus logros y avances son los dos tipos de contenidos que los trabajadores mejor valoran, con un 4,75 sobre 5 .

En relación con los canales por los que los empleados reciben la información que les llega de la cooperativa, el mejor valorado es el Cara a Cara con un 4,42 sobre 5, seguido de los Medios de Comunicación, con un 4,12, y el correo electrónico y las reuniones, ambas con un 4 de media.

Al igual que los socios encuestados, los empleados valoran WhatsApp (75\%) y las reuniones $(50 \%)$ como los mejores canales de comunicación para mantenerse informados de los nuevos hechos y acontecimientos. Caso similar es el de las redes sociales en las que los empleados tienen perfiles activos, ya que al igual que los socios, WhatsApp con un $87 \%$ y Facebook (62\%) son las dos redes en las que tienen mayor presencia.

En contraposición a los socios, los empleados comparten menos información de la cooperativa en sus redes sociales. Un $63 \%$ dice hacerlo frente al $37 \%$ que asegura 
que no. Por otra parte, un $75 \%$ de los empleados encuestados reconoce sentirse informado a la hora de compartir información en redes, frente al $13 \%$ que dice no estarlo.

A la pregunta de si solicitan información a una fuente oficial de la cooperativa cuando quiere contestar a un mensaje en las redes y considera que no dispone de la información necesaria, el $87 \%$ de los empleados reconoce hacerlo.

En relación con los canales de comunicación, al 100\% de los encuestados les gustaría disponer de uno o varios canales por los que realizar consultas y encontrar información. Los canales preferidos para desarrollar esa función son WhatsApp y la página web.

El $100 \%$ de los empleados encuestados afirma haber encontrado en las redes sociales, o en algún medio de comunicación, informaciones falsas o rumores que tienen la intención de dañar la reputación de la cooperativa. El $88 \%$ reconoce que consultó al departamento de comunicación antes de ofrecer ninguna respuesta frente al $12 \%$ restante que asegura que rebatió por su cuenta aquellas informaciones malintencionadas.

Este tipo de desinformaciones afecta de manera directa en los empleados. El $100 \%$ reconocen que les preocupa porque daña la imagen y la reputación de la cooperativa, junto al $75 \%$ que reconoce que le afecta porque se siente parte de la sociedad. De igual forma, a un $37 \%$ de los encuestados les preocupa la parte económica en la que pueda verse dañada la cooperativa.

Al hablar de bulos y desinformación, el $75 \%$ de los empleados dice saber reconocer una noticia falsa, para ello es importante analizar la fecha, la fiabilidad de la fuente y el origen del que procede la información.

Además, al igual que los socios, el $75 \%$ de los empleados consideran muy importante contar con una ayuda que proceda de la cooperativa y que les facilite diferenciar este tipo de desinformaciones, el $25 \%$ restante lo considera bastante importante. Debido a este factor, el $50 \%$ de los empleados encuestados estaría dispuesto a consultaría un blog dirigido desde el departamento de comunicación, que contase con información, formación y tutoriales sobre posibles bulos, rumores e informaciones que dañan la imagen de la sociedad, y sobre cómo responder y conocer el posicionamiento de DCOOP. Destaca que un $13 \%$ asegura que lo consultaría siempre y cuando se le avisara de que los contenidos se actualizan.

Por último, y al igual que a los socios, se les pregunto a los empleados cómo valorarían de 1 a 5 , siendo 5 la nota más alta, las diferentes acciones a llevar a cabo desde el departamento de comunicación para afrontar cualquier crisis en redes sociales y combatir las noticias falsas y los rumores sobre la sociedad. Formar a los socios y empleados para que sepan afrontar esas situaciones y contar con un protocolo definido internamente e implicando a los empleados serían, a juicio de los trabajadores, las dos mejores formas de afrontar las crisis en las redes sociales y combatir las noticias falsas y los rumores sobre la cooperativa. 


\section{CONCLUSIONES}

El fin de este trabajo se centraba, por un lado, en analizar los pasos que se habían dado hasta ahora en materia de verificación y actuación ante desinformaciones y bulos, desde gabinetes de comunicación. Y, por otro, se pretendía establecer un protocolo de actuación desde los diferentes gabinetes de empresas e instituciones para hacer frente a los posibles casos de bulos, rumores y desinformaciones que se publican y difunden con el objetivo final de dañar la reputación de una sociedad.

Así, se considera que contar con un plan de comunicación se establece como pieza fundamental en toda estrategia de una empresa y además, este debe estar alineado con el modelo de negocio de esta. Dentro de este, contar con un plan de crisis que recoja además una estrategia de transparencia y proactiva resulta imprescindible para alcanzar una comunicación eficaz. Así se desprende de las respuestas de socios y empleados, que además, muestran su disposición para implicarse en esta tarea en la estrategia en las redes sociales.

Gracias a la realización de los cuestionarios, se observa que la reputación de la empresa u organización debe reforzarse en el seno de la entidad, y mantenida a través de la comunicación interna, incentivando, además, la escucha activa en las redes sociales para identificar cualquier hecho desinformativo o rumor sobre una empresa. Esto será una de las claves en la lucha contra la desinformación. Los empleados y los socios pueden convertirse, en este caso, en los verdaderos ojos y oídos de la empresa en los medios digitales, así como en la voz que responda y complete en algunos casos la posición de la propia empresa.

En cuanto a las diferentes desinformaciones que se analizaron en el estudio de caso, destaca que todas tuvieron una gran repercusión debido al papel que juegan Internet y las redes sociales. Tal y como afirmaba Burkhardt (2017), los contenidos engañosos o manipulados siempre han existido, pero las conocidas como fake news alcanzan una difusión mucho mayor debido a este panorama digital en el que todo el mundo está interconectado.

Asimismo, y coincidiendo con lo que Losada et., al (2020) recogen, las desinformaciones consiguen una mayor difusión que otros tipos de contenidos por la forma en la que apelan a las emociones. Así es que, como se observa en el estudio de caso, las diferentes desinformaciones publicadas eran compartidas un gran número de veces gracias a los sentimientos de enfado y engaño que proyectaban. Es importante destacar que, al igual que Tapia Frade et al., (2011) concluyen, el papel de la web como canal principal de comunicación, esta debe ser transparente y estar actualizada de forma periódica y con contenido relevante para todos sus públicos. De forma que, en situaciones de crisis, como la vivida por la cooperativa en 2018, el contacto directo y permanente con los públicos de interés mitigue significativamente el daño que esa crisis pueda provocar.

Gracias al desarrollo de este trabajo se pone de manifiesto, tal y como señala diferentes expertos consultados, que cada vez es más necesario establecer un equipo dedicado a la monitorización de la imagen de una compañía, junto a la creación de un protocolo de actuación con las herramientas necesarias para desmentir las posibles 
desinformaciones, bulos y rumores que se difundan y que dañen la reputación de la empresa o institución.

Conviene resaltar la necesidad de informar de manera permanente a los diferentes públicos, de forma más constante, sobre temas como la marcha económica de la cooperativa, los logros que esta alcanza y tener conocimiento de la versión de la sociedad ante cualquier bulo o desinformación que se difunda sobre ella ayuda a cualquier empresa a enfrentarse a cualquier crisis basada en rumores y desinformación.

Para ello, es importante hacer uso de las redes sociales, ya que coincidiendo con lo que aseguran Pablo et al., (2021) establecer una estrategia para cada una de ellas es fundamental a la hora de enfrentarse a una crisis. Además, el papel que juegan las redes dentro de la comunicación hoy es fundamental para lograr una conexión entre las empresas e instituciones y sus stakeholders.

Tal y como señalaban Rodríguez-Pérez (2021) y Lara (2021), los distintos públicos de interés de la entidad o institución serán los mejores portavoces de la sociedad cuando tengan que hablar en sus propios grupos. Esta relación se logra si se ofrece información fiable de forma que ellos mismos tengan sus propios argumentos.

Es importante tener en cuenta que, el eje principal de todo plan de comunicación y estrategia comunicativa se centra en la comunicación permanente. Ser proactivos y transparentes en todo lo que se comunica es muy importante para mantener la reputación y evitar, sobre todo en los casos de crisis, los daños que sobre ella puedan recaer. En el caso de la cooperativa de segundo grado DCOOP, sería un buen complemento para su estrategia comunicativa alinear el modelo de negocio con la comunicación que esta transmite a sus socios y, por supuesto, a sus clientes

Como elemento principal para vehicular la estrategia de comunicación, la web se establece como el punto principal de su relación con los públicos, tanto internos como externos. Debe ser el medio de comunicación por excelencia de la organización. Es por ello por lo que, incorporar dentro de la página web corporativa elementos como el informe anual y el de responsabilidad social corporativa, la agenda con los diferentes eventos, las apariciones de la cooperativa en medios, videos promocionales, tutoriales y encuestas, making of de los diferentes procesos de elaboración y forma de trabajar de la cooperativa, etc.; de forma que todo esto fomente la creación de una comunidad virtual, basada en el intercambio planificado, hasta donde se pueda, de un flujo permanente de mensajes entre la entidad y todos sus grupos de interés. Se pueden crear espacios dedicados especialmente a cada uno de estos grupos y otros generales.

En cuanto a las redes sociales, no podemos obviar la importancia que estas juegan en el nuevo panorama digital. Las redes sociales permiten llegar al cliente y establecer una relación con él, pero, del mismo modo, si esas redes se implementan con carácter corporativo se convierten un punto a favor de la comunidad virtual, en este caso, de la cooperativa con sus stakeholders. Esto implica que la empresa debe reforzar y planificar su presencia en estos canales con más visión estratégica, tanto en la difusión como en la escucha activa, convirtiendo estos espacios en canales con 
información de calidad, transparentes, al servicio de los usuarios, los socios, los empleados y los clientes.

Otra de las propuestas a realizar, y que coincide con el objetivo de este trabajo, es la creación de una Unidad de Verificación de Noticias y Bulos. Para ello se debe de implantar un protocolo en el que es muy importante el papel proactivo de la empresa o institución, basado en la escucha activa y permanente de los grupos de interés, así como en la formación y alfabetización digital para los socios y empleados, fundamentalmente. Asimismo, se deben de definir los pasos que se deben de realizar cuando se detectan desinformaciones que pueden dañar la imagen de las empresas o instituciones; que se resumen en la monitorización e identificación constante de rumores y desinformación sobre la organización y entidades similares para estar en alerta, definir el tipo de crisis y de desinformación o rumor para prevenir y anticiparnos a sus consecuencias, comprobar la repercusión de estos, medir los tiempos y la magnitud de la respuesta.

Para poder desarrollar este protocolo de forma eficaz, es necesario que exista una formación permanente entre los empleados y empleadas y socios y socias de la cooperativa, de manera que, cada vez sea mayor el número de personas que sepan identificar una desinformación y cuál es la forma adecuada de combatirla.

Para poder completar los puntos de este protocolo se debe de contar con las herramientas necesarias que permitan llevar a cabo la verificación de los contenidos. Algunas de ellas pueden ser Crowdtangle, Tineye o DomainBigData.

\section{BIBLIOGRAFÍA}

Aced, C. (2013). El perfil del dircom 2.0. En Cuadernos de Comunicación Evoca, Comunicación Corporativa: Al otro lado de la información. (pp. 17-22). Evoca Comunicación e Imagen.

Anuario de la Comunicación. (2014). Tendencias mundiales de la comunicación corporativa. Asociación de Directivos de Comunicación. Dircom.

Boyle, M., \& Schmierbach, M. (2015). Applied Communication Research Methods. Getting Started as a Researcher. Routledge. https://doi.org/10.4324/9781315718644

Burkhardt, J. M. (2017). History of Fake News. Library Technology Reports, 53(8), 133.

Callejo, G. J. (2002). Observación, entrevista y grupo de discusión: el silencio de tres prácticas de investigación. Revista Española Salud Pública, 76(2), 409-422.

Egelhofer, J. L., \& Lecheler, S. (2019). Fake news as a two-dimensional phenomenon: a framework and research agenda. Annals of the International Communication Association, 43(2), 97-116. https://doi.org/10.1080/23808985.2019.1602782 
Espinosa, F. T. (2021). El aprendizaje basado en objetos como estrategia para la enseñanza de la historia en Educación Primaria. Cuadernos de profesorado, 14(28), 44-56.

Estudio de comunicación. (2020). Empleados comprometidos, eficaces. Estudio de comunicación.

Fita, J. (2002). Comunicación en programas de crisis. Ediciones Gestión 2000.

Forni, P. (2010). Los estudios de caso: Orígenes, cuestiones de diseño y sus aportes a la teoría social. Miríada: Investigación en Ciencias Sociales, 3(5), 61-80.

Fundación Gabo. (2021). El Periodismo ante la desinformación. Fundación Gabo.

García, T. (2003). El cuestionario como instrumento de investigación/evaluación. Página Del Proyecto de Apoyo Para Profesionales de La Formación (PROMETEO) de La Junta de Andalucía, 28.

Hernández, R., Baptista, P., \& Hernández, C. (2004). Metodología de la Investigación. McGraw-Hill Interamericana, 533.

Humprecht, E. (2019). Where 'fake news' flourishes: a comparison across four Western democracies. Information Communication and Society, 22(13), 19731988. https://doi.org/10.1080/1369118X.2018.1474241

Jiménez-Chaves, V. (2012). El estudio de caso y su implementación en la investigación. Revista Internacional de Investigación En Ciencias Sociales, 8(1), 141-150.

Jiménez, E. (2013). Management Crisis in Social Networks. Revista Orbis, 24, 116131.

Kawulich, B. B. (2005). FORUM: qualitative social research. La observación participante como método de recolección de datos 1. Fqs, 6(2), 32.

Losada, R. (2016). La labor del Dircom en la comunicación de crisis. Más Poder Local, 29, 12-13.

Losada, D. J. C. (2019). Comunicación de crisis en un mundo conectado. Editorial: EDIUOC.

Losada, D. J. C., Rodríguez, F. L. y Paniagua, R. F. J. (2020). Comunicación gubernamental y emociones en la crisis del Covid-19 en España. Revista Latina de Comunicación Social, 78, 23-40. https://www.doi.org/10.4185/RLCS-2020-1467

Macnamara, J. (2016) Organizational listening: Addressing a major gap in public relations theory and practice, Journal of Public Relations Research, 28 (3- 4), 146169. https://doi.org/10.1080/1062726X.2016.1228064 
Macnamara, J., \& Gregory, A. (2018) Expanding Evaluation to Progress Strategic Communication: Beyond Message Tracking to Open Listening. International Journal of Strategic Communication, 469-486. https://doi.org/10.1080/1553118X.2018.1450255

Magallón-Rosa, R. (2018). Nuevos formatos de verificación. El caso de Maldito Bulo en Twitter. Sphera Publica, 1(18), 41-65.

Merino-Poyo, M. J. (2016). Tesis Doctorl. Comunicación y crisis: un plan estratégico. http://eprints.ucm.es/36040/1/T36709.pdf

Meneses, J., \& Rodríguez, R. (2011). El cuestionario y la entrevista. Construcció d'instruments d'investigació en e-Learning, 5-31.

Mitroff, I., \& Pearson, C. M. (2002). Cómo gestionar una crisis. Guía para mejorar la preparación frente a una crisis. Ediciones Gestión 2000.

Molina, C. J. P., Magallón, R. R., \& Paniagua, R. F. J. (2020). Desinformación y factchecking en las elecciones argentinas de 2019. El caso de la iniciativa Reverso. Revista de estilos de aprendizaje, 13(26), 33-49.

Monje, A. C. A. (2011). Metodología de la investigación cuantitativa y cualitativa. Guía didáctica. Universidad Surcolombiana, 1-216.

Morris, A. (2018). The what and why of in-depth interviewing. A Practical Introduction to InDepth Interviewing, 1-16. https://doi.org/10.4135/9781473921344.n1

Nyilasy, G. (2019) Fake news: When the dark side of persuasion takes over, International Journal of Advertising, 38(2), 336-342. https://doi.org/10.1080/02650487.2019.1586210

Pablo, P., Dueñas, M., \& Carmona, D. G. (2021). Comunicación digital en las cooperativas españolas. 193-225. https://doi.org/10.7203/CIRIEC-E.101.17638

Piñuel, R. J. (2002). Epistemología, métodos y técnicas del análisis de contenido. Sociolinguistic Studies, 3(1), 1-42.

Regan, E. O. (2008). Capítulo cuarto Redes sociales: una herramienta para la comunicación estratégica. Uso de las redes sociales en la comunicación de la defensa Evangeline $O^{\prime}$ Regan. El Mundo, 83-102. https://doi.org/10.1558/sols.v3i1.1

Reyes, T. (1999). Métodos cualitativos de investigación: Los grupos focales y el estudio de caso. Fórum Empresarial, 4(2), 74-87. https://doi.org/10.33801/fe.v4i2.2913

Rodríguez-Fernández, L. (2019). Desinformación: retos profesionales para el sector de la comunicación. El profesional de la información, 28(3), 1-11. https://doi.org/10.3145/epi.2019.may.06 
Rodríguez-Fernández, L. (2019). Desinformación y comunicación organizacional: estudio sobre el impacto de las fake news. Revista Latina de Comunicación Social, 1714 -1728.http://www.revistalatinacs.org/074paper/1406/89es.html

Rodríguez, G. D., \& Valldeoriola, R. J. (2009). Metodología de la investigación. Universidad Oberta de Catalunya

Rodríguez, P. C. (2019). No diga fake news, di desinformación: una revisión sobre el fenómeno de las noticias falsas y sus implicaciones. Comunciación, revista científica, 40, 65-74. http://dx.doi.org/10.18566/comunica.n40.a05

Ruiz, O. J. I. (2012.) Teoría y práctica de la investigación cualitativa. Universidad de Deusto.

Saura, P. P., \& García-García, F. (2012). La comunicación de crisis como elemento clave de la comunicación empresarial. Revista ICONO14. Revista Científica de $\begin{array}{llll}\text { Comunicación } y & \text { Tecnología Emergentes, } & \text { 8(2), } & 42 .\end{array}$ https://doi.org/10.7195/ri14.v8i2.245

Taddicken, M., \& Wolff, L. (2020). 'Fake News' in Science Communication: Emotions and Strategies of Coping with Dissonance Online. Media and Communication, 8(1), 206 -217. https://doi.org/10.17645/mac.v8i1.2495

Tandoc, E. C., Wei, L. Z., \& Ling, R. (2018). Defining “Fake News”, Digital Journalism, 6(2), 137-153. https://doi.org/10.1080/21670811.2017.1360143

Tandoc, Jr. E. C., Thomas, R. J., \& Bishop, L. (2021). What Is (Fake) News? Analyzing News Values (and More) in Fake Stories. Media and Communication, 9(1), 110-119. https://doi.org/10.17645/mac.v9i1.3331

Tapia-Frade, A., Gómez-Nieto, B., \& Díaz, C. Ó. (2011). La página web como herramienta para la retroalimentación en las organizaciones: el caso de las ONGs españolas. Doxa Comunicación. Revista Interdisciplinar de Estudios de $\begin{array}{lllll}\text { Comunicación } y & \text { Ciencias } \quad \text { Sociales, } & 12,86 .\end{array}$ https://doi.org/10.31921/doxacom.n12a3

Ufarte-Ruiz, M. J., Peralta-García, L., \& Murcia-Verdú, F. J. (2018). Fact checking: un nuevo desafío del periodismo. El profesional de la información, 27(4), 733-741. https://doi.org/10.3145/epi.2018.jul.02

Villafane, J. (1999). La gestión profesional de la imagen corporativa. Pirámide. Madrid: Pirámide

Vosoughi, S., Roy, D., \& Aral, S. (2019). The spread of true and false news online. Science, 359, 1146-1151.

Wardle, C. (2017, 14 de marzo). First Draft. https://firstdraftnews.org/latest/noticiasfalsas-es-complicado/ 
Weare, C., Lin, W., \& Lin, W. (1987). Social Science Computer Review. Social Science Computer Review, 5(4), 608.

https://doi.org/10.1177/089443938700500420

Yin, R. (1994). Investigación sobre estudios de casos. Diseño y metología. Applied Social Research Methods Series, 5, 1-35. http://www.polipub.org/documentos/YIN ROBERT.pdf

\section{AUTORES:}

\section{Paula Vázquez Almendros}

Estudiante de Periodismo en la Universidad de Málaga y actualmente realiza prácticas profesionales, como analista de desinformación y de redes sociales en el departamento de comunicación de Dcoop.

\section{Francisco Javier Paniagua Rojano}

Profesor titular de Periodismo en la Universidad de Málaga, experto en comunicación corporativa y redes sociales. Actualmente es coordinador de la sección "Comunicación y estrategia organizacional" de la Asociación Española de Investigación de la Comunicación y ha sido director de comunicación de la Federación Andaluza de Municipios y Provincias (2000-2003) y la Universidad Internacional de Andalucía (2011-2014).

Orcid ID: https://orcid.org/0000-0001-7376-4536 